\title{
Long-term viral brain-derived neurotrophic factor delivery promotes spasticity in rats with a cervical spinal cord hemisection
}

\author{
Karim Fouad ${ }^{1}$, David J. Bennett ${ }^{2}$, Romana Vavrek ${ }^{1}$ and Armin Blesch ${ }^{3,4}$ \\ ${ }^{1}$ Rehabilitation Medicine, University of Alberta, Edmonton, AB, Canada \\ 2 University of Alberta, Edmonton, AB, Canada \\ ${ }^{3}$ Spinal Cord Injury Center, University Hospital Heidelberg, Heidelberg, Germany \\ ${ }^{4}$ University of California, San Diego, La Jolla, CA, USA
}

Edited by:

Renée Morris, The University of New

South Wales, Australia

\section{Reviewed by:}

Catherine Gorrie, University of

Technology, Australia

Lorne M. Mendell, Stony Brook

University, USA

*Correspondence:

Karim Fouad, Rehabilitation Medicine, Centre for Neuroscience, University of Alberta, 3-87 Corbett Hall, Edmonton, AB T6G 2G4, Canada e-mail: karim.fouad@ualberta.ca
We have recently reported that rats with complete thoracic spinal cord injury (SCl) that received a combinatorial treatment, including viral brain-derived neurotrophic factor (BDNF) delivery in the spinal cord, not only showed enhanced axonal regeneration, but also deterioration of hind-limb motor function. By demonstrating that BDNF over-expression can trigger spasticity-like symptoms in a rat model of sacral $\mathrm{SCl}$, we proposed a causal relationship between the observed spasticity-like symptoms (i.e., resistance to passive range of motion) and the over-expression of BDNF. The current study was originally designed to evaluate a comparable combined treatment for cervical $\mathrm{SCl}$ in the rat to improve motor recovery. Once again we found similar signs of spasticity involving clenching of the paws and wrist flexion. This finding changed the focus of the study and, we then explored whether this spasticity-like symptom is directly related to the over-expression of BDNF by administering a BDNF antagonist. Using electromyographic measurements we showed that this treatment gradually diminished the resistance to overcome forelimb flexion in an acute experiment. Thus, we conclude that neuro-excitatory effects of chronic BDNF delivery together with diminished descending control after $\mathrm{SCl}$ can result in adverse effects.

Keywords: spinal cord injury, BDNF, combined treatment, TrkB-Fc, EMG

\section{INTRODUCTION}

Axonal regeneration in the adult mammalian central nervous system is inhibited by numerous factors (1) impeding the development of effective treatments for brain or spinal cord injuries (SCI). Consequently, it is likely that repair of the injured spinal cord by axonal regeneration and plasticity will require a combined treatment approach. Several combinatorial strategies have been pursued with a wide range of outcomes [e.g., Ref. (2-5)]. These experiments are not only technically demanding, but also challenging in the interpretation of the results, considering the unpredictable effects of promoting neurite outgrowth and possible interactions between treatment components $(6,7)$.

One prominent molecule that has been the subject of many studies in spinal cord repair is brain-derived neurotrophic factor (BDNF). BDNF not only has neuro-protective, regeneration, and plasticity promoting effects but also neuro-excitatory properties and binds with high affinity to the TrkB receptor and with lower affinity to 75 [reviewed in Ref. (8)]. As such, BDNF is a prominent candidate for combined treatment approaches [e.g., Ref. $(6,9,10)]$. However, due to the broad range of its effects, the consequences of BDNF delivery can be complex. For example, we observed that a combinatorial treatment involving BDNF (achieved by direct viral vector injection and bone marrow stromal cell grafts genetically modified to express BDNF) promoted spasticity in rats with a cervical hemisection. Because the combined treatment also promoted axonal regeneration and plasticity, the origin of the observed spasticity remains unclear (11). We could, however, provide an indirect explanation by demonstrating that the over-expression of BDNF could promote the development of hyperreflexia in tail muscles in a sacral model of SCI (11). Yet, it remains unclear whether this effect of BDNF was caused by its neuro-excitatory properties or through an augmentation of neuroplasticity and/or regenerative growth. However, this was not the original question of the current study. We initially set out to test a combined treatment that addressed multiple limitations for neurite growth following cervical SCI. The focus of the analysis shifted when severe spasticity-like symptoms in the injured forelimb became evident, which led us to focus on determining the cause of spasticity. To address this question we administered a BDNF antagonist into the spinal cord of rats that showed spasticity-like symptoms, and these were gradually reduced by this treatment.

\section{MATERIALS AND METHODS ANIMALS}

Adult female Fischer 344 rats (Charles River, 180-220 g) were group housed and kept at $12 / 12 \mathrm{~h} \mathrm{light/dark}$ cycle with ad libitum water. The study was approved by the Animal Care and Use Committee for Health Sciences of the University of Alberta, and complies with the guidelines of the Canadian Council for Animal Care. 


\section{LESION SURGERY AND TREATMENT}

Rats were anesthetized (using Hypnorm $0.16 \mathrm{mg} / \mathrm{kg}$; Vetapharma, Leeds, UK; and Midazolam $2.5 \mathrm{mg} / \mathrm{kg}$; Sandoz, Canada; diluted in sterile water) and mounted into a stereotaxic frame (Kopf Instruments), and the spinal cord between C5 and C6 was exposed by performing a laminectomy of half of the C5 segment. The spinal cord was lesioned using a microsuction pipette and a spring scissor. A lateral hemisection lesion was performed ipsilateral to the preferred paw as determined by a forelimb reaching task. A small gap was created to ensure lesion completeness and provide space for the cell graft. The dura was sealed with a thin agarose film (Sigma) and fibrin glue (Baxter, USA), overlying muscles were sutured and the skin was stapled. Rats were placed on a heating pad until they were awake and received buprenorphine $(0.03 \mathrm{mg} / \mathrm{kg})$ as analgesic and saline over the next 2 days.

\section{Preparation of cell grafts to provide a tissue bridge for regenerating axons}

Fibroblasts were isolated from skin biopsies of Fischer 344 rats and cultivated in D'MEM/10\% FBS with antibiotics. Cells were transduced with retroviral vectors to express BDNF as described by Lu et al. $(11,12)$ or NT-3 (13) and selected for G418 resistance. BDNF and NT-3 expression was measured in vitro, in $24 \mathrm{~h}$ cell culture supernatants by ELISA as described $(14,15)$. BDNF-transduced fibroblasts expressed $27 \mathrm{ng} / 10^{6}$ cells $/ 24 \mathrm{~h}, \mathrm{NT}-3$ transduced cells expressed $134 \mathrm{ng} / 10^{6}$ cells $/ 24 \mathrm{~h}$. For grafting, BDNF and NT-3 expressing cells were mixed $1: 1$ and $2-3 \mu l$ were injected into the lesion site using a 5- $\mu$ l Hamilton syringe at a concentration of $2.5 \times 10^{4}$ cells $/ \mu \mathrm{l}$.

\section{scAAV vector preparation and injections}

Self-complementary adeno-associated viral vectors (scAAV) were generated as described by Lu et al. (11). In order to attract regenerative growth out of the graft into the caudal spinal cord, BDNF, and NT-3 (and GFP as control) transfected scAAV vectors $(1.3 \mu \mathrm{l} / \mathrm{site}$ ) were injected $1.25,2.5$, and $4 \mathrm{~mm}$ caudal to the lesion on the side ipsilateral to the lesion through pulled glass capillaries using a Picospritzer II similar to Lu et al. (11). The injection volume was divided over a depth of 1 and $1.5 \mathrm{~mm}$.

One week post-lesion surgery, cell grafts and viral vector injection surgeries were performed in all lesioned animals in the following groups:

Group 1 is a CONTROL group used to demonstrate the ability of self-complementary adeno-associated virus (scAAV) vectors to successfully infect spinal neurons. This group did not receive a cell graft, only PBS, followed by injections of $4.3 \times 10^{11} \mathrm{vg} / \mathrm{ml} \mathrm{scAAV}$ vectors expressing Green Fluorescent Protein (scAAV2-GFP) at $1.25,2.5$, and $4 \mathrm{~mm}$ caudal to the lesion; $n=16$.

Group 2, scAAV-BDNF group received an injection of PBS in the lesion site, followed by $4.3 \times 10^{11} \mathrm{vg} / \mathrm{ml}$ scAAV-BDNF injections at $1.25,2.5$, and $4 \mathrm{~mm}$ caudal to the lesion to serve as trophic support for regenerating fibers; $n=5$.

Group 3, the GRAFT/BDNF/NT-3 or FULL treatment group, received a fibroblast cell graft (expressing BDNF and NT-3) into the lesion site to provide a permissive cellular matrix that allows axonal growth, and a trophic stimulus to stimulate axonal growth.
This group also received injections of a mixture of scAAV2 BDNF (f.c. $4.3 \times 10^{11} \mathrm{vg} / \mathrm{ml}$ ), scAAV2-NT-3 (f.c. $4.3 \times 10^{11} \mathrm{vg} / \mathrm{ml}$ ), and scAAV2-GFP (f.c. $4 \times 10^{10} \mathrm{vg} / \mathrm{ml}$ ) mixed with $20 \mathrm{U} / \mathrm{ml} \mathrm{ChABC}$ (Seigaku, USA) caudal to the lesion $(1.25,2.5$, and $4 \mathrm{~mm})$. The neurotrophic mixture is intended to provide trophic stimulus beyond the lesion and ChABC degrades growth inhibitory proteoglycans in the scar tissue (3). In addition, this group also received a subcutaneous injection of the phosphodiesterase (PDE) inhibitor, Rolipram, (AG Scientific, San Diego, CA, USA; $3 \mathrm{ml}$ of $3 \mathrm{mg} / \mathrm{kg}$ in $\%$ DMSO and saline) once per day for 14 days following the lesion. This drug elevates cAMP levels in the CNS and augments regeneration-related gene expression in injured neurons (16); $n=18$.

Group 4, the GRAFT/scAAV-NT-3 group received a fibroblast cell graft expressing BDNF and NT-3 into the lesion site, injections of scAAV expressing NT-3 and GFP only, mixed with ChABC caudal to the lesion and subcutaneous injections of rolipram; $n=7$. Group 5, the GRAFT/ChABC group received a fibroblast cell graft expressing BDNF and NT-3 into the lesion site and ChABC injections $1.25,2.5$, and $4 \mathrm{~mm}$ caudal to the lesion, followed by daily rolipram administration; $n=5$.

\section{BEHAVIORAL TESTING}

\section{Single-pellet grasping task}

This test was performed as described previously $(17,18)$. In order to motivate rats to grasp for sugar pellets $(45 \mathrm{mg}$, banana flavor; TestDiet, Richmond, CA, USA) in the task, restricted amounts of food were allowed directly after the daily training session $(10 \mathrm{~g} / \mathrm{rat} /$ day $)$. A transparent Plexiglas chamber $(30 \mathrm{~cm} \times 36 \mathrm{~cm} \times 30 \mathrm{~cm})$ with a narrow opening in the front wall and an attached shelf outside $(2 \mathrm{~cm}$ distance from the inside of the front wall, $3 \mathrm{~cm}$ above the floor) was used. Pellets were placed at a distance such that the rats had to grasp them with their preferred forelimb and could not reach them with their tongue. The preferred paw was determined for each rat during the first few training sessions. Successful grasps were scored over a 10-min period only when a rat was able to grasp the presented pellet, bring it to its mouth and eat it. Pre-operative baseline values were obtained after a 4 -week training period (five times per week, 10 min per rat). Postoperative rehabilitative training of the injured forelimb started 1 week after graft implantation surgery ( 2 weeks post-lesion).

\section{Forelimb placement}

To further assess forelimb function, all rats were scored over a 4min period in a Plexiglas open field according to Martinez et al. (19), with scores ranging from 0 (no function) to 20 (no deficit). Behavioral components were categorized from global fore- and hind-limb movements to antero-posterior coordination, which included weight support, fine distal positioning, and stepping abilities. Nine weeks post-lesion, asymmetries in spontaneous forelimb use were evaluated by placing individual rats into a clear Plexiglas cylinder $(24 \mathrm{~cm}$ high/19 $\mathrm{cm}$ inner diameter). A mirror was placed underneath the cylinder at an angle to facilitate the videotaping of the rat's vertical exploratory activity in the cylinder (Figure 1). Ten rearing movements were recorded in each test session. Each forepaw placement on the wall of the cylinder was scored during a rearing movement. If the affected forepaw was 

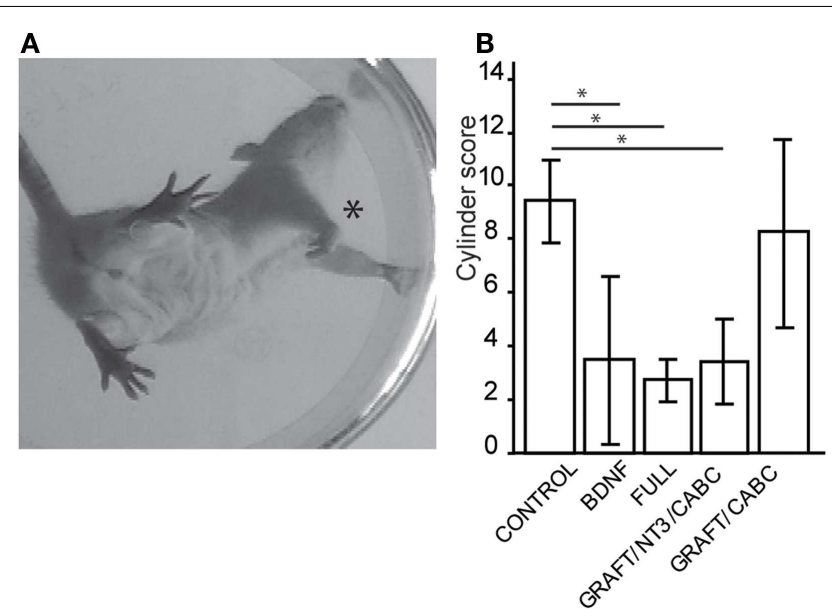

FIGURE 1 | Treatments involving BDNF promoted exaggerated wrist flexion and affected forelimb use. Signs of wrist flexion became apparent as early as 2 weeks after treatment (A). By 9 weeks post-injury the cylinder exploration task indicated that animals in the FULL treatment group and the scAAV-BDNF only group had significantly reduced use of the injured forelimb $\left(^{*}\right)$ when compared to control animals (B). Error bars show average $\pm \mathrm{SE} ;{ }^{*} p<0.05,{ }^{*} p<0.005,{ }^{* *} p<0.0005$. plantar on contact with the wall of the cylinder, the number of contacts was multiplied by two, if only the dorsal surface of the paw was placed on the wall, the number of contacts was multiplied by one and if the paw did not contact the wall a score of 0 was assigned, thus allowing for a maximum score of 20 .

\section{Grip strength}

The grip strength of the forelimbs (or in other words, the force needed to overcome wrist and digit flexion when actively or passively holding on to a bar) was assessed 9 weeks post-lesion by measuring the maximum force (in gram) exerted by each forelimb, with a Grip Strength Meter apparatus (Columbus Instruments, $\mathrm{OH}$, USA, Figure 2). For the final values, four readings for both paws were obtained over three different trials and averaged for post-operative analysis.

\section{Sensory test}

Forelimb withdrawal latency to a heat stimulus was measured prior to injury (baseline) and 9 weeks post-lesion using methods previously described (20). Briefly, the rats were placed on a glass plate over a light box, and a radiant heat stimulus (Ugo Basile) was applied by aiming a beam of light onto the plantar surface of the paw of each forelimb through the glass plate. The light beam was turned off automatically when the rat lifted the limb, allowing the measurement of time between the start of the light beam and the paw withdrawal. Five minutes were allowed between three trials, baseline values were averaged for each limb and compared to post-injury values.

\section{ELECTROPHYSIOLOGY}

Electromyographic (EMG) recordings of flexor muscles of the injured paw were carried out prior to perfusion. Animals were anesthetized with 5\% isoflurane gas anesthesia for induction and
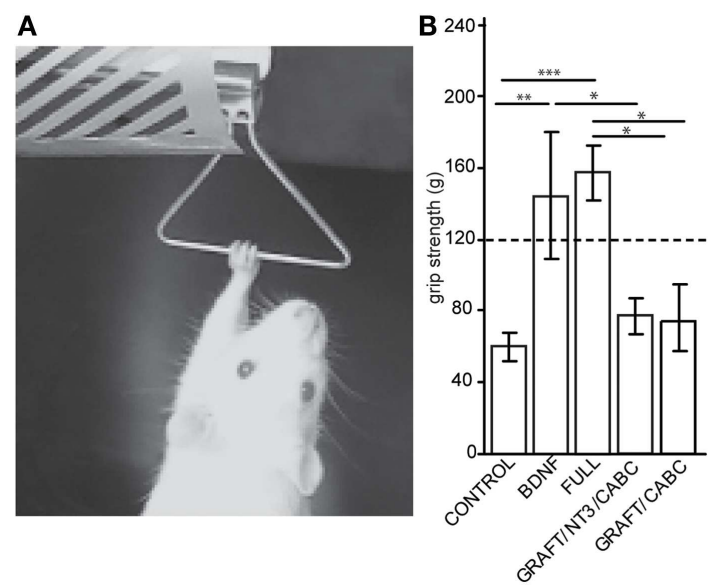

FIGURE 2 | Brain-derived neurotrophic factor expression increases wrist flexion: using an apparatus designed to measure grip strength (A) we compared measurements between control animals and the rats receiving scAAV-BDNF only or the FULL treatment (B). When compared with the control group, a significant increase in "grip" strength was observed in both the scAAV-BDNF and the FULL treatment groups at 9 weeks post treatment. The dashed line indicates a normal grip strength value. Error bars show average $\pm \mathrm{SE} ;{ }^{* *} p<0.01,{ }^{* * *} p<0.001$.

$1.5 \%$ for maintenance of anesthesia and placed into a stereotaxic frame. The spinal cord surrounding the hemisection lesion was exposed. To record EMG responses during manual wrist extension, two electrodes with exposed tips (Teflon coated wire; A-M Systems, Carlsborg, WA, USA) were inserted into wrist flexors of the forelimb ipsilateral to the lesion. The EMG signal was amplified (Grass, Astro-Med Inc., West Warwick, RI, USA), digitized ( $5 \mathrm{kHz}$, Digidata 1322A; Axon instruments, Foster City, CA, USA), and filtered $(30-300 \mathrm{~Hz})$ as described earlier (17). The muscle responses were recorded under anesthesia before and 10, 20, and $30 \mathrm{~min}$ following injection of the TrkB antagonist, TrkB-Fc ( $1 \mu \mathrm{l}$ of $0.2 \mathrm{mg} / \mathrm{ml}$; Sigma) or saline into the spinal cord lesion site.

The following animals were selected for EMG analysis: (i) three rats without signs of spasticity (one from the CONTROL group and two from the FULL treatment group) received a TrkB-Fc injection. (ii) Four rats with spasticity (one from the scAAV-BDNF, two from the FULL treatment group and one from GRAFT/NT3) received a saline injection only. (iii) Ten rats with spasticity (two from scAAV-BDNF and eight from FULL treatment group) received TrkB-Fc injection.

Electromyographic responses were recorded, and the burst duration was measured using Axoscope software (Axoscope 9.0.1.16; Molecular Devices, Sunnyvale, CA, USA). The traces were rectified and the average burst amplitude was quantified and then multiplied by the burst duration using Microsoft Excel.

The animals were euthanized immediately following the EMG recordings with pentobarbital (Euthanyl, Bimeda-MTC; $70 / 100 \mathrm{mg}$ body weight) and transcardially perfused with phosphate-buffered saline, followed by $4 \%$ paraformaldehyde (PFA) in $0.1 \mathrm{M}$ phosphate buffer, $\mathrm{pH}$ 7.4. The spinal cord was removed, post-fixed in $4 \%$ PFA overnight at $4^{\circ} \mathrm{C}$ and 
cryo-protected in 30\% sucrose over 2 days. The cervical enlargement encompassing the lesion site (C2-6) was embedded in Tissue Tek (Sakura Finetek USA Inc., Torrance, CA, USA) and frozen in 2-methyl-butane at $-60^{\circ} \mathrm{C}$.

\section{HISTOLOGY}

Spinal cords were cryosectioned in the horizontal plane at $25 \mu \mathrm{m}$ and mounted in eight series onto Fisherbrand slides (Fisher Scientific, Ottawa, ON, Canada). To evaluate the lesion site, sections were counterstained with $0.1 \%$ Cresyl Violet and dehydrated in increasing alcohol concentrations, cleared with xylene, and coverslipped with Permount (Fisher Scientific). Lesion sizes were reconstructed from $\mathrm{C} 2$ to $\mathrm{C} 6$ spinal cord tissue by analyzing every fourth section through the dorso-ventral plane of the spinal cord by using central canal and gray/white matter interphases as landmarks. From these reconstructions, the percent cross-sectional surface area of the damaged tissue was measured using Image J (NIH) software. Animals with lesions deviating from a complete hemisection by $>10 \%$ were removed from this study.

Immuno-histochemistry of serotonergic (5-HT) fibers was carried out on every second horizontal section encompassing the lesion site using rabbit anti 5-HT antibody (S5545, Sigma-Aldrich, Oakville, ON, Canada). In brief, following a 10\% normal goat serum (NGS, Vector Labs, Burlingame, CA, USA) blocking step, the slides were incubated in 1:1000 dilution of primary antibody containing $1 \%$ NGS overnight at $4^{\circ} \mathrm{C}$. The slides were then washed in TBS before an overnight incubation at $4^{\circ} \mathrm{C}$ in the secondary antibody (Vector Labs, Burlingame, CA, USA). Application of ABC and $\mathrm{DAB}$ solutions according to manufacturer's recommendations followed (Vector Laboratories, Burlingame, CA, USA). After three washing steps of $10 \mathrm{~min}$ in TBS, the slides were serially dehydrated with alcohol, cleared with xylene, and coverslipped in Permount (Sakura Finetek USA, Torrance, CA, USA).

The density of 5-HT fibers rostral and caudal of the lesion was quantified from images taken at $400 \times$ using a Leica light microscope. Images were saved as 8-bit gray-scale TIFF files and imported into Image J software version $1.43 \mathrm{q}(\mathrm{NIH})$, where threshold values were adjusted. Selected threshold values were kept constant for all images to standardize the amount of background included in quantification. Integrated density measurement, representing the sum of the values of the pixels in an image, was averaged from five different images.

\section{STATISTICS}

To determine statistical differences between treated and untreated animals, Student's $t$-test and a one-way ANOVA with Tukey's honestly significant difference post hoc test (Prism, V 4.0; GraphPad, San Diego, CA, USA) were used. All results and figures are presented as means \pm SE of the mean. Statistical significance is stated for $p$ values $<0.05$.

\section{RESULTS \\ FUNCTIONAL RECOVERY}

The post-lesion recovery process was overshadowed by the appearance of signs of spasticity in the forelimb ipsilateral to the lesion. The first signs involved clenching of the paw and especially pronounced wrist flexion that appeared 2 weeks post-injury. Post hoc analysis indicated that these signs of spasticity occurred in animals in all treatment groups. However, by 6 weeks, only $12 \%$ of rats (2 out of 16) in the control treatment group showed abnormal wrist flexion and clenching of the paw. This stood in contrast to $60 \%$ ( 3 out of 5 ) of rats that were injected with scAAV-BDNF and $72 \%$ ( 13 out of 18 ) of rats belonging to the FULL treatment group (involving cell grafts expressing BDNF and NT-3 in the lesion site and scAAV-BDNF expression caudal to lesion) and three out of seven rats $(43 \%)$ in the GRAFT/NT-3 group which received the cell grafts expressing BDNF and NT-3 in the lesion site and scAAVNT-3 only expression caudal to lesion. In the, GRAFT/ChABC group, consisting of cell grafts expressing BDNF and NT-3 and ChABC injection, only one out of five rats $(20 \%)$ displayed signs of spasticity. These effects made further functional testing difficult. Rats with these signs of spasticity were unable to plantar step or to grasp for food pellets. Consequently, because most animals were unable to retrieve pellets, the single-pellet task had to be abandoned entirely. Although rats in all groups attempted to reach for pellets, a significant decline in the attempt rate was apparent in the treated groups when compared to their baseline attempt rate measurements (FULL treatment group: 15.4\%, scAAV-BDNF group: 35\%, GRAFT/NT-3 group: 56.7\%; GRAFT/ChABC group: $25.7 \%$, and control group: $86.7 \%$ ).

A test capable of quantitatively assessing the deficits in hand/wrist function was the cylinder test (Figure 1A). When exploring the wall with their paws, rats showing signs of spasticity did not use the affected limb to explore, significant deficits were observed in the group receiving scAAV-BDNF only ( $3.5 \pm 1.3)$, in the group receiving graft expressing BDNF/NT-3 and scAAV-NT3 expressing vectors $(3.4 \pm 1.6)$, as well as in the FULL treatment group $(2.7 \pm 0.9)$ when compared to control group $(9.4 \pm 1.5)$ or the graft expressing BDNF/NT-3 and ChABC treated group $(8.25 \pm 3.4$; Figure 1B).

When quantifying the forelimb deficits using a forelimb score [Ref. (19); a score of 0 indicates no forelimb function and 20 indicates no deficit] rats in the group receiving scAAV-BDNF only performed somewhat worse than control treated rats $(9.2 \pm 0.6$ vs. 11.2 \pm 0.8 ); however this difference was not found to be statistically significant. When controls were compared to the rats receiving the FULL treatment with markedly more cells expressing $\mathrm{BDNF}$ (due to the graft) a significant decrease in the performance was found ( $7.3 \pm 0.5 ; p=0.0008$; data not shown).

A test that dramatically illustrates the inability to open the paw and the rigidity of the wrist flexors in these rats is the grip strength test (Figure 2A). By sliding the paw over the bar of the force sensor, the paw basically "hooked" onto the bar and the animal could be pulled away from the sensor in order to measure the force needed to overcome flexion and to release the bar. When comparing these forces between the groups for the unaffected arm (contralateral to the lesion), no differences were found. This is of interest considering that a potential neuro-excitatory effect of BDNF could spread over the entire spinal cord. Because the values were comparable between all groups, an average value from all animals (118.2 $\pm 4 \mathrm{~g})$ is indicated in Figure 2B as "normal" grip strength.

When testing the affected limb we found that control animals with hemisection lesion (and no treatment) usually exert a moderate to weak resistance when being pulled away from the 
bar. The force necessary before releasing/sliding the affected paw over the bar was significantly lower than that for the unaffected $\operatorname{paw}(s)(60.1 \pm 31.5$ vs. $118.2 \pm 4 \mathrm{~g} ; p=0.022)$. In contrast, rats with signs of spasticity were hardly able to release the bar resulting in higher values than those in the unaffected paw and significantly higher than the control treated rats on the affected side (scAAVBDNF vs. Control $p=0.0057$; FULL vs. Control $p=0.0001$; scAAV-BDNF vs. GRAFT/NT-3 $p=0.048$; FULL vs. GRAFT/NT$3 p=0.001$; FULL vs. GRAFT/ChABC $p=0.012$ ). Rats receiving only scAAV-BDNF were statistically indiscernible from those receiving the full treatment $(144.2 \pm 35.6$ vs. $157.2 \pm 15.6)$ as both had abnormally high "grip" strength. Whereas the GRAFT/NT-3 treated group $(76.7 \pm 10.1 \mathrm{~g})$ was not different from the CONTROL $(60.1 \pm 31.5 \mathrm{~g})$, or the GRAFT/ChABC treated group (73.5 $\pm 10.2 \mathrm{~g})$.

In summary, during normal activities, the majority of rats receiving scAAV-BDNF only or in combination with other treatments were unable to extend their wrist or open the digits of their paw. Further, the average force needed to overcome the wrist flexion was significantly increased.

This unexpected treatment-induced motor deficit, likely related to BDNF over-expression by cell grafts and spinal scAAV injection, did not allow the continuation of the originally planned experiment, and consequently, the focus shifted to the possible role of BDNF in the development of spasticity. Therefore, histological results are only reported for serotonergic fibers because of serotonin's neuro-excitatory properties and potential role in the development of spasticity.

Because of the involvement of BDNF in nociception (21), the Hargreaves apparatus was used to analyze the possible treatment effect on the latency of injured forelimb withdrawal in response to a painful stimulus pre and post treatment. Only two groups, FULL and the GRAFT/NT-3, showed a significant reduction in latency of withdrawal of their preferred forelimb ( $p$ value: 0.016 and 0.038 , respectively), indicating thermal hypersensitivity, in response to thermal stimulation when compared to baseline latency values (FULL: 9.0 vs. $11.4 \mathrm{~s}$, GRAFT/NT-3 9.5 vs. $11.4 \mathrm{~s}$ ). Other treatment groups did not show any significant change in withdrawal latency following lesion (data not shown). These results do indicate that within our experiment there was no obvious link between changes in nociception and the occurrence of spasticity-like symptoms. The lack of effects on thermo-sensitivity in some of the groups where BDNF was over-expressed could be based on various factors including effects of other treatment components or insufficient BDNF levels.

\section{ANIMAL WEIGHTS}

Because earlier findings indicate that BDNF reduces weight gain in rats $(22,23)$, decreased weight in the groups of animals receiving scAAVs expressing $\mathrm{BDNF}$ or a combination of grafts expressing BDNF and scAAVs expressing BDNF, might be one potential indicator for adverse effects of increased BDNF levels (Figure 3A).

When we compared the animal weights at the end of the experiment (i.e., 9 weeks post-injury) between the control rats that received a lesion and GFP expressing scAAV vectors $(207 \pm 1.7 \mathrm{~g})$ and those that received only scAAV-BDNF $(201 \pm 1.1 \mathrm{~g})$, we found

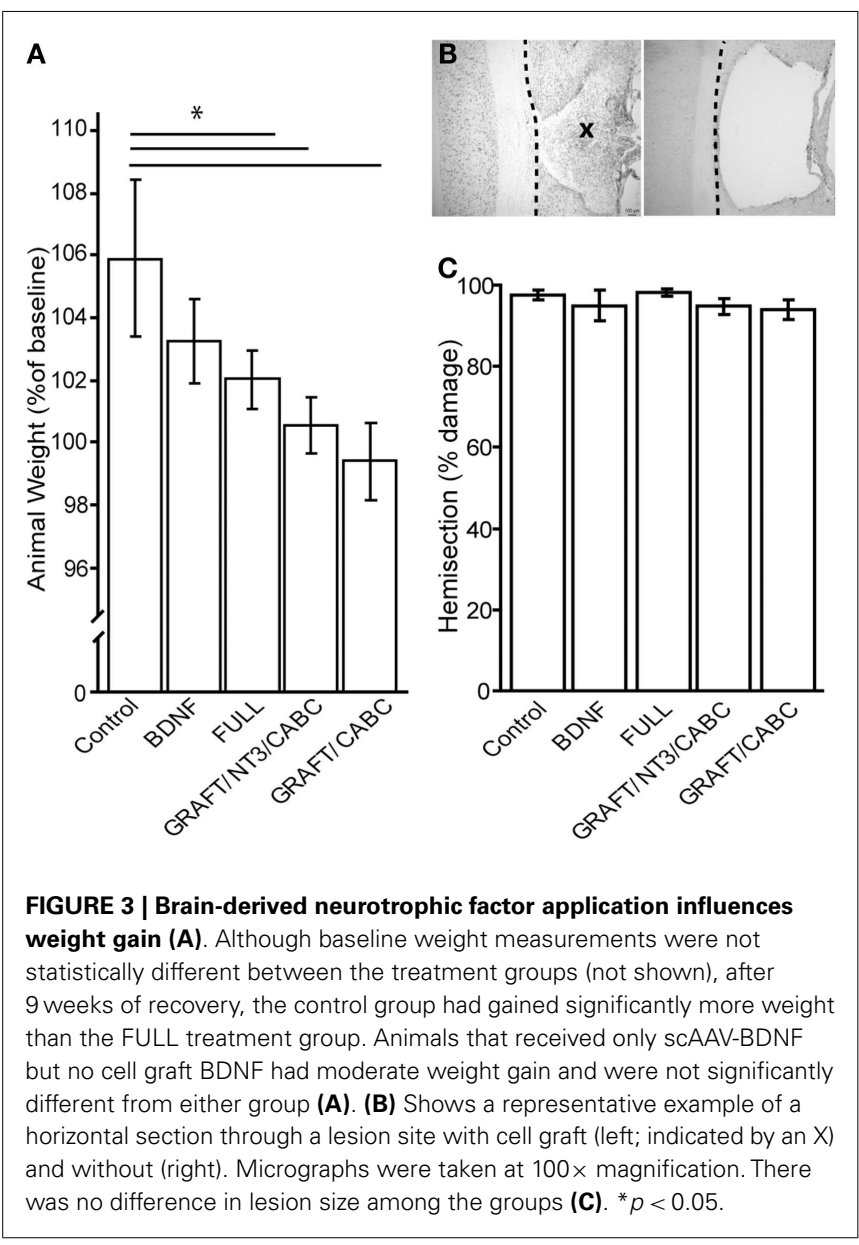

a small, non-statistically significant decrease in weight gain. Rats that received the FULL treatment (which entails, among others, BDNF and NT-3 expressing cell grafts in addition to the injection of scAAV-BDNF) showed a significant difference (192 $\pm 2.3 \mathrm{~g})$. GRAFT/NT-3 and GRAFT/ChABC treated group was not statistically different from any other treatment groups. However, it has to be kept in mind that this weight loss might have also been influenced by other treatment components such as rolipram injections in addition to the chronic BDNF over-expression by either the fibroblast cell graft or scAAV vectors.

\section{LESION SIZES}

Lesion sizes were analyzed from reconstruction of serial sections (Figures 3B,C). Following our exclusion criteria, animals with lesions deviating from a complete hemisection by $>10 \%$ were removed from this study $(n=4)$; a comparison of the lesion sizes between the groups showed no statistical difference between either group. The control group had only $2.2 \%( \pm 1.1)$ tissue spared in comparison to $4.1 \%( \pm 2.8)$ in the scAAV-BDNF only group, 1.1\% $( \pm 1.4)$ in the FULL treatment group, $3.7( \pm 0.01)$ in the GRAFT/NT-3 group, or $4.12( \pm 0.01)$ in the GRAFT/ChABC group. Thus, variations in lesion size were only minor, and differences in functional outcome can therefore confidently be attributed to the different treatments. 


\section{SPROUTING OF SEROTONERGIC FIBERS}

Nine weeks after SCI, the density of 5-HT positive fibers both immediately rostral and caudal to the lesion site was quantified in order to visualize and compare the possible role of 5-HT fiber sprouting in the observed spasticity following SCI (Figure 4A).

The integrated density of 5-HT fibers was compared among all groups (Figure 4B). The highest values for 5-HT were observed in the GRAFT/NT-3 group ( $2923 \pm 307)$ which is statistically significant when compared to the control group $(2148 \pm 117)$ and the scAAV-BDNF only group $1621 \pm 418$ ( $p=0.0330,0.0424$, respectively). The average density value in the FULL treatment group was $2354 \pm 212$ and $2423 \pm 337$ in GRAFT/ChABC (not significant to controls).

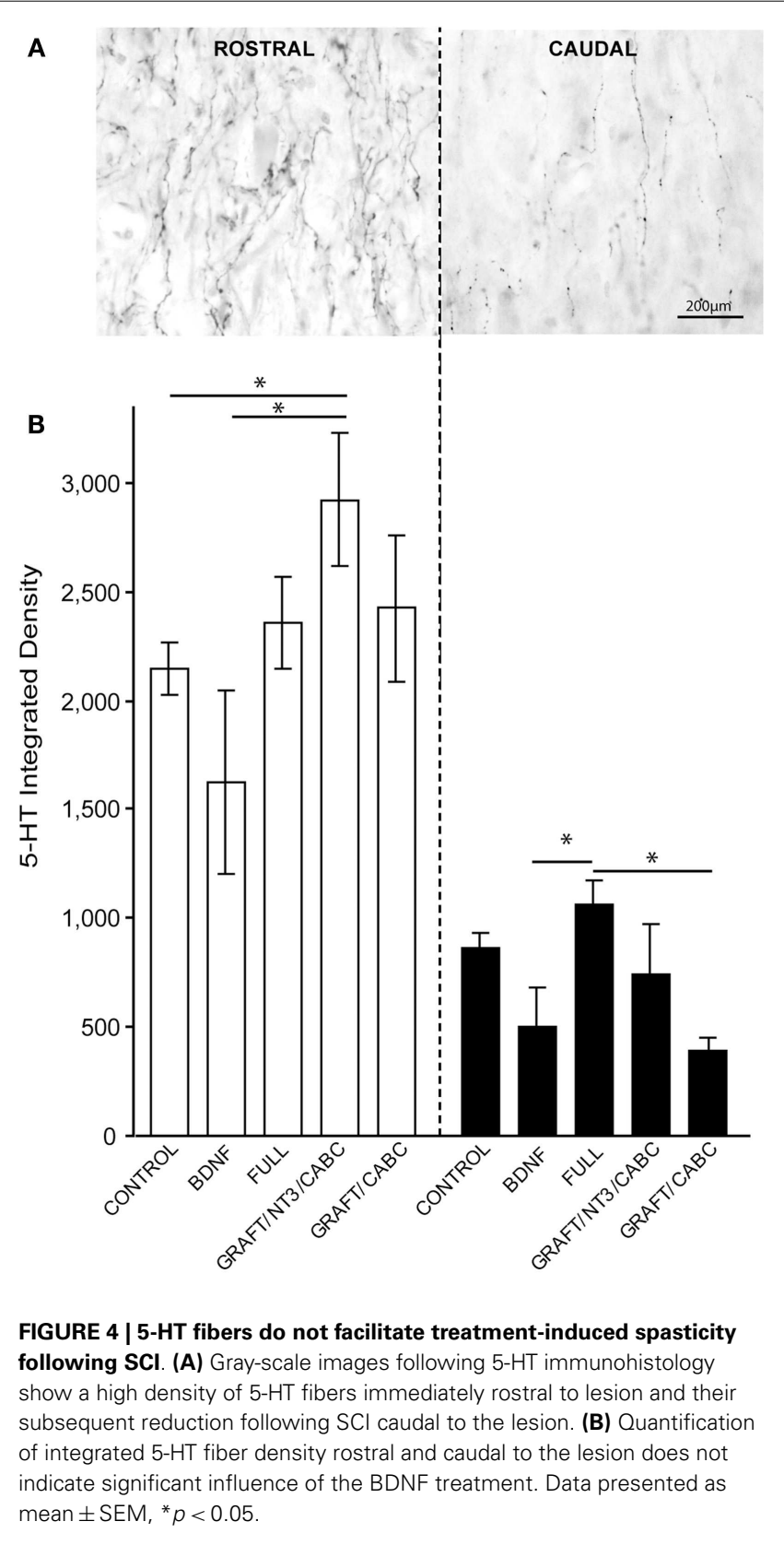

Caudal to the lesion, 5-HT density was reduced and even though the FULL treatment group contained significantly more fibers than the scAAV-BDNF only group $(p=0.034 ; 1059 \pm 116$ vs. $503 \pm 178$; respectively), neither group was statistically different from the control group ( $863 \pm 68)$. The FULL treatment group was however, significantly different from the GRAFT/ChABC group $(1059 \pm 116$ vs. $395 \pm 52 ; p=0.0339)$, but not different from GRAFT/NT-3 group ( $1059 \pm 116$ vs. $739 \pm 229)$. Thus, it appears unlikely that differences in the availability of serotonin contributed to the increase in spasticity of the treated groups.

\section{ANTAGONIZING BDNF}

In order to investigate the underlying mechanisms of the observed increase in spasticity-like symptoms of animals that received BDNF treatment either by scAAV vector expression or in combination with grafts expressing BDNF, we quantified the muscle activity elicited by overcoming the wrist flexion before and after the spinal injection of the BDNF antagonist TrkB-Fc.

Before injection of TrkB-Fc into the lesion site, wrist flexion was manually overcome three times in a row with an interval of about $3 \mathrm{~s}$, which was repeated every $10 \mathrm{~min}$ following the TrkB-Fc injection for a total of $30 \mathrm{~min}$. When this experiment was performed in rats without signs of spasticity $(n=3)$, there was no resistance to the stretch and no EMG activity was detected before or during the extension of the wrist into a horizontal position (i.e., $180^{\circ}$ ) even before TrkB-Fc was injected. Therefore, for this experiment only rats that demonstrated resistance and an EMG response in flexor muscles to stretching of the wrist (i.e., displayed spasticity-like symptoms) were included.

When averaging the rectified EMG from all three stretches and comparing this value to values measured at 10 and $20 \mathrm{~min}$ following a spinal saline injection, we found in four animals with spasticity-like symptoms (one from the scAAV-BDNF, two from the FULL treatment group, and one from GRAFT/NT-3 group) only an insignificant decrease in EMG response over time (Figure 5). Only after $30 \mathrm{~min}$, a significant decline of $11 \%$ was observed.

In contrast, in 10 animals with spasticity-like symptoms (two scAAV-BDNF and eight of the FULL treatment group) that received the BDNF antagonist injection, the EMG response dropped by $51 \pm 9.8 \%$ starting as early as $10 \mathrm{~min}$ after TrkB-Fc injection and continued to decline to $64 \pm 8.4 \%$ at $20 \mathrm{~min}$ and $80 \pm 6.6 \%$ at $30 \mathrm{~min}$. The difference in the stretch induced average and rectified EMG amplitude at each time point was statistically significant between saline and TrkB-Fc injected rats (Figure 5).

Interestingly, a significant effect of TrkB-Fc was also found in one out of two control treated rats that showed severe wrist flexion/spasticity, at 20 and 30 min post injection $(p=0.024$ and 0.036 , respectively).

\section{DISCUSSION}

Brain-derived neurotrophic factor is a prominent neurotrophic factor with a long history and a variety of effects. Such effects include the modulation of cell survival (ranging from protection to cell death), the enhancement of neurite outgrowth and axonal regeneration, the promotion of myelination, as well as the modulation of synaptic transmission, the post-injury immune 

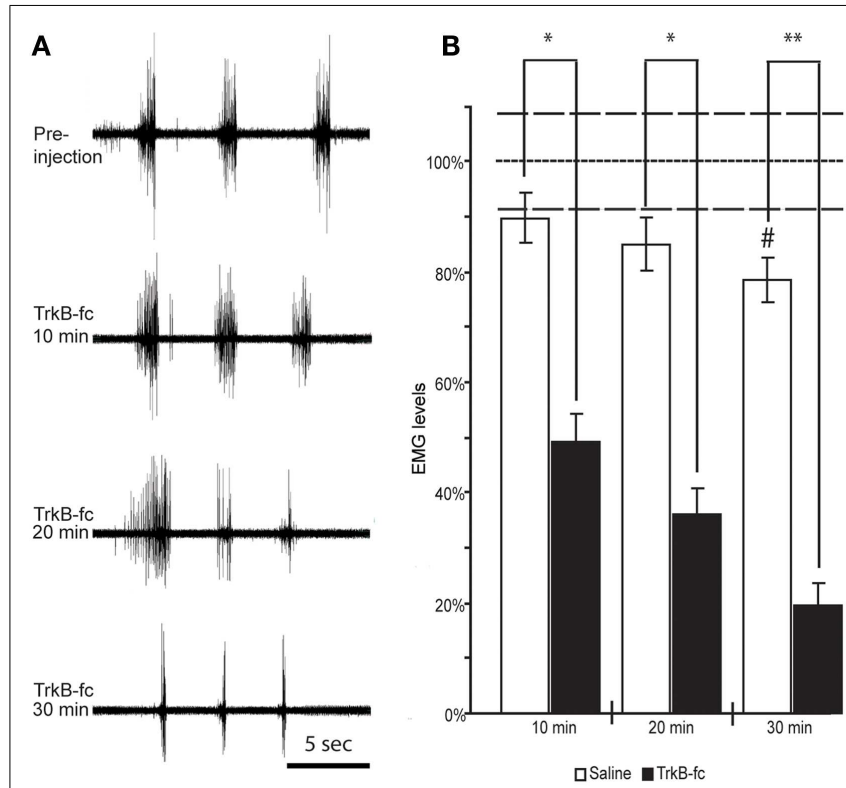

FIGURE 5 | Spinal BDNF antagonist reduces resistance to overcome wrist flexion in animals that exhibit spasticity-like symptoms. EMG recordings from wrist flexor muscles of the forelimb ipsilateral to the lesion before and at three different time points following TrkB-Fc injection show a gradual decline in stretch-evoked EMG responses. Note the relative inactivity in the recording before and in between the stretch-evoked bursts (A). When quantifying the EMG response and normalizing it to the values found in control animals under anesthesia only (did not receive Saline or TrkB-Fc injections; dotted line) we found a significant drop in EMG response at $10 \mathrm{~min}$, and a continuous decline over the next 20 min when TrkB-FC infusion was compared to saline injections (B). "Indicates a significant reduction of EMG response at 30 min post saline injection when compared to control animals that were under anesthesia only (dotted line; $p=0.0286$ ). White columns represent rats that received saline, black columns represent rats that received the BDNF antagonist. Bars show means \pm SEM; ${ }^{*} p<0.05$. ${ }^{*} p<0.005$. Dashed lines indicate the SEM for control anesthesia (dotted line).

response and neuronal excitability [reviewed in Ref. (8)]. Not surprisingly, BDNF also frequently makes it onto the list of potential SCI treatments [e.g., Ref. $(11,24-30)]$. Considering the broad effects of BDNF, it is also not surprising that the mechanisms by which BDNF influences functional outcomes is sometimes difficult to discern. Understanding the mechanism by which a treatment influences functional recovery (or decline) is more challenging when several treatments are combined. A good example of this can be found in a recent report by $\mathrm{Lu}$ and colleagues (11), where a combined treatment not only resulted in enhanced plasticity of various descending fiber systems (e.g., serotonergic fibers) and axonal regeneration, but also in an undesired effect (i.e., increased occurrence of spasticity). Obviously it is of utmost importance to explore whether the increased spasticity was due to treatment-mediated augmentation of neurite growth and plasticity and/or possibly through an acute neuro-excitatory effect of $\operatorname{BDNF}(31,32)$.

Another challenge of using BDNF for treating SCI is its wellestablished role in affecting sensory and nociceptive pathways in the spinal cord (21) and its ability to modulate glutamate receptors and sensitization $(33,34)$. Thus, a possible link between the observed spasticity-like symptoms found in the present study and neuropathic pain could be suggested. Yet, in this study no consistent change was found in thermo-sensitivity and it is unlikely that the signs of spasticity are related to increased pain. For example, it could be assumed that when assessing the grip strength, increased pain sensitivity would result in a withdrawal rather than an increase in grip strength.

Another potential mechanism involved in promoting spasticity could be aberrant serotonergic sprouting and the subsequent increased levels of serotonin, which could increase neuronal excitability $(35,36)$. In the current study, we did not find significant differences in the serotonergic innervation between the groups and demonstrate that a BDNF antagonist reduces spasticity within minutes. This indicates that an acute neuro-excitatory effect of permanent BDNF expression underlies the increase in signs of spasticity. The relatively fast effect of the TrkB antagonist also indicates that, although baseline activity in the flexed muscle was very low, a contracture [shortening of the muscle; Ref. (37)] was not involved in the observed spasticity-like symptoms.

It has to be kept in mind that spasticity-like symptoms were also observed in a small percentage of rats with spinal hemisection that received only GFP expressing viral vectors. It appears as though the continuous BDNF over-expression exacerbated this naturally occurring process by decreasing the threshold for the development of spasticity. Curiously, the application of the BDNF antagonist also reduced the spasticity in one control treated rat, indicating that BDNF signaling might also be involved in post-injury regulation of neuronal excitability. Indeed, increases in BDNF expression after SCI have been reported (38-40), which can contribute to increased neuronal excitability by reducing the amount of current required to reach threshold [i.e., rheobase; Ref. $(41,42)$ ].

There are many possible mechanisms by which BDNF can exert its excitatory effects. For example, BDNF may increase calcium and sodium influx through TrkB signaling (43). Furthermore, activation of the TrkB-PLC pathway may raise the membrane potential through calcium-mediated channel opening (44). BDNF has also been reported to down-regulate the potassium-chloride co-transporter KCC2 (45), which down-regulates the inhibitory influence of gamma-aminobutyric acid (GABA) receptors. The most rapid and potent excitatory effects of BDNF, however, have been shown to involve Nav1.9, on a time scale similar to the action of glutamate (32).

Considering its neuro-excitatory properties, it is not surprising that over-expression of BDNF can also promote beneficial effects without promoting neuronal survival or regeneration. For example, Boyce et al. (41), showed that the injection of BDNF expressing vectors improved locomotor function in rats with complete spinal cord transections.

Effects of BDNF on synaptic connectivity have been described in dissociated cultures of early embryonic hippocampal neurons, where it increased the number of functional synaptic connections (46). At the same time, studies of long-term effects of BDNF in dissociated cortical cultures have suggested that BDNF decreases neuronal firing rate by reducing the strength of all excitatory inputs onto a given neuron (47). Bolton et al. (48) found that actions of 
BDNF underlying the increase in activity, involved enhancement of both excitatory and inhibitory synaptic transmission in parallel, but via distinct cellular mechanisms.

In the current study, it can only be speculated which neuronal population(s) responded to the BDNF antagonist, since the stretch induced spasticity-like reflex was decreased. From earlier studies $(31,49)$ it is likely that motoneurons play a major role in the development of spasticity. Generally speaking, following the administration of the BDNF antagonist we found a decline in EMG amplitude (see Figure 5 at $20 \mathrm{~min}$ ) and a decline in burst duration in parallel to the decline in spasticity-like symptoms. This is in line with the idea that after SCI spasticity is related to the re-occurrence of plateau potentials in motoneurons allowing activity beyond the time of depolarization $(49,50)$.

In conclusion, although the permanent expression of BDNF using viral vectors may well promote certain aspects of recovery through neuro-excitatory mechanisms, the potential side effects would caution such application and point to the use of regulated expression systems to control BDNF expression (14). The general involvement of the TrkB receptor in the development of spasticity warrants further exploration.

\section{ACKNOWLEDGMENTS}

Supported by grants from Wings for Life to Karim Fouad and NIH/NINDS (NS054883), Wings for Life, International Foundation for Research in Paraplegia and International Spinal Research Trust to Armin Blesch. We would like to thank Dr. M. Tuszynski for his contributions in the design of the study.

\section{REFERENCES}

1. Gervasi NM, Kwok JC, Fawcett JW. Role of extracellular factors in axon regeneration in the CNS: implications for therapy. Regen Med (2008) 6:907-23. doi:10.2217/17460751.3.6.907

2. Coumans JV, Lin TT, Dai HN, MacArthur L, McAtee M, Nash C, et al. Axonal regeneration and functional recovery after complete spinal cord transection in rats by delayed treatment with transplants and neurotrophins. J Neurosci (2001) 21(23):9334-44.

3. Fouad K, Schnell L, Bunge MB, Schwab ME, Liebscher T, Pearse DD. Combining Schwann cell bridges and olfactory-ensheathing glia grafts with chondroitinase promotes locomotor recovery after complete transection of the spinal cord. J Neurosci (2005) 25(5):1169-78. doi:10.1523/JNEUROSCI.3562-04.2005

4. Houle JD, Tom VJ, Mayes D, Wagoner G, Phillips N, Silver J. Combining an autologous peripheral nervous system "bridge" and matrix modification by chondroitinase allows robust, functional regeneration beyond a hemisection lesion of the adult rat spinal cord. J Neurosci (2006) 26(28):7405-15. doi:10.1523/JNEUROSCI.1166-06.2006

5. Kadoya K, Tsukada S, Lu P, Coppola G, Geschwind D, Filbin MT, et al. Combined intrinsic and extrinsic neuronal mechanisms facilitate bridging axonal regeneration one year after spinal cord injury. Neuron (2009) 64(2):165-72. doi:10.1016/j.neuron.2009.09.016

6. Bretzner F, Liu J, Currie E, Roskams AJ, Tetzlaff W. Undesired effects of a combinatorial treatment for spinal cord injury-transplantation of olfactory ensheathing cells and BDNF infusion to the red nucleus. Eur J Neurosci (2008) 28(9):1795-807. doi:10.1111/j.1460-9568.2008.06462.x

7. García-Alías G, Barkhuysen S, Buckle M, Fawcett JW. Chondroitinase ABC treatment opens a window of opportunity for task-specific rehabilitation. $\mathrm{Nat} \mathrm{Neu}$ rosci (2009) 12(9):1145-51. doi:10.1038/nn.2377

8. Weishaupt N, Blesch A, Fouad K. BDNF: the career of a multifaceted neurotrophin in spinal cord injury. Exp Neurol (2012) 238(2):254-64. doi:10.1016/ j.expneurol.2012.09.001

9. Bamber NI, Li H, Lu X, Oudega M, Aebischer P, Xu XM. Neurotrophins BDNF and NT-3 promote axonal re-entry into the distal host spinal cord through Schwann cell-seeded mini-channels. Eur J Neurosci (2001) 2:257-68. doi:10.1046/j.1460-9568.2001.01387.x

10. Li C, Zhang X, Cao R, Yu B, Liang H, Zhou M, et al. Allografts of the acellular sciatic nerve and brain-derived neurotrophic factor repair spinal cord injury in adult rats. PLoS One (2012) 7(8):e42813. doi:10.1371/journal.pone.0042813

11. Lu P, Blesch A, Graham L, Wang Y, Samara R, Banos K, et al. Motor axonal regeneration after partial and complete spinal cord transection. J Neurosci (2012) 32(24):8208-18. doi:10.1523/JNEUROSCI.0308-12.2012

12. Lu P, Blesch A, Tuszynski MH. Neurotrophism without neurotropism: BDNF promotes survival but not growth of lesioned corticospinal neurons. J Comp Neurol (2001) 436(4):456-70. doi:10.1002/cne.1080

13. Grill RJ, Blesch A, Tuszynski MH. Robust growth of chronically injured spinal cord axons induced by grafts of genetically modified NGF-secreting cells. Exp Neurol (1997) 148(2):444-52. doi:10.1006/exnr.1997.6704

14. Blesch A, Tuszynski MH. Transient growth factor delivery sustains regenerated axons after spinal cord injury. J Neurosci (2007) 27(39):10535-45. doi:10.1523/JNEUROSCI.1903-07.2007

15. Taylor L, Jones L, Tuszynski MH, Blesch A. Neurotrophin-3 gradients established by lentiviral gene delivery promote short-distance axonal bridging beyond cellular grafts in the injured spinal cord. J Neurosci (2006) 26(38):9713-21. doi:10.1523/JNEUROSCI.0734-06.2006

16. Nikulina E, Tidwell JL, Dai HN, Bregman BS, Filbin MT. The phosphodiesterase inhibitor rolipram delivered after a spinal cord lesion promotes axonal regeneration and functional recovery. Proc Natl Acad Sci U S A (2004) 101(23):8786-90. doi:10.1073/pnas.0402595101

17. Krajacic A, Ghosh M, Puentes R, Pearse DD, Fouad K. Advantages of delaying the onset of rehabilitative reaching training in rats with incomplete spinal cord injury. Eur J Neurosci (2009) 29(3):641-51. doi:10.1111/j.1460-9568.2008. 06600.x

18. Whishaw IQ, Pellis SM, Gorny B, Kolb B, Tetzlaff W. Proximal and distal impairments in rat forelimb use in reaching follow unilateral pyramidal tract lesions. Behav Brain Res (1993) 56(1):59-76. doi:10.1016/0166-4328(93)90022-I

19. Martinez M, Brezun JM, Bonnier L, Xerri C. A new rating scale for open-field evaluation of behavioral recovery after cervical spinal cord injury in rats. $\mathrm{J} \mathrm{Neu}$ rotrauma (2009) 26(7):1043-53. doi:10.1089/neu.2008.0717

20. Hargreaves K, Dubner R, Brown F, Flores C, Joris J. A new and sensitive method for measuring thermal nociception in cutaneous hyperalgesia. Pain (1988) 32(1):77-88. doi:10.1016/0304-3959(88)90026-7

21. Kerr BJ, Bradbury EJ, Bennett DL, Trivedi PM, Dassan P, French J, et al. Brain-derived neurotrophic factor modulates nociceptive sensory inputs and NMDA-evoked responses in the rat spinal cord. J Neurosci (1999) 19(12): 5138-48.

22. Bariohay B, Roux J, Tardivel C, Trouslard J, Jean A, Lebrun B. Brain-derived neurotrophic factor/tropomyosin-related kinase receptor type B signaling is a downstream effector of the brainstem melanocortin system in food intake control. Endocrinology (2009) 150(6):2646-53. doi:10.1210/en.2008-1184

23. Fouad K, Vavrek R, Cho S. A TrkB antibody agonist promotes plasticity following cervical spinal cord injury in adult rats. J Neurotrauma (2010). doi:10.1089/neu.2009.1116

24. Kobayashi NR, Fan DP, Giehl KM, Bedard AM, Wiegand SJ, Tetzlaff W. BDNF and NT-4/5 prevent atrophy of rat rubrospinal neurons after cervical axotomy, stimulate GAP-43 and Talphal-tubulin mRNA expression, and promote axonal regeneration. J Neurosci (1997) 17(24):9583-95.

25. McTigue DM, Horner PJ, Stokes BT, Gage FH. Neurotrophin-3 and brainderived neurotrophic factor induce oligodendrocyte proliferation and myelination of regenerating axons in the contused adult rat spinal cord. J Neurosci (1998) 18(14):5354-65.

26. Jakeman LB, Wei P, Guan Z, Stokes BT. Brain-derived neurotrophic factor stimulates hindlimb stepping and sprouting of cholinergic fibers after spinal cord injury. Exp Neurol (1994) 154(1):170-84. doi:10.1006/exnr.1998.6924

27. Liu Y, Kim D, Himes BT, Chow SY, Schallert T, Murray M, et al. Transplants of fibroblasts genetically modified to express BDNF promote regeneration of adult rat rubrospinal axons and recovery of forelimb function. J Neurosci (1999) 19(11):4370-87.

28. Hiebert GW, Khodarahmi K, McGraw J, Steeves JD, Tetzlaff W. Brain-derived neurotrophic factor applied to the motor cortex promotes sprouting of corticospinal fibers but not regeneration into a peripheral nerve transplant. JNeurosci Res (2002) 69(2):160-8. doi:10.1002/jnr.10275 
29. Lu P, Jones LL, Tuszynski MH. BDNF-expressing marrow stromal cells support extensive axonal growth at sites of spinal cord injury. Exp Neurol (2005) 20191(2):344-60. doi:10.1016/j.expneurol.2004.09.018

30. Mantilla CB, Gransee HM, Zhan WZ, Sieck GC. Motoneuron BDNF/TrkB signaling enhances functional recovery after cervical spinal cord injury. Exp Neurol (2013) 247:101-9. doi:10.1016/j.expneurol.2013.04.002

31. Boulenguez P, Liabeuf S, Bos R, Bras H, Jean-Xavier C, Brocard C, et al. Downregulation of the potassium-chloride co-transporter KCC2 contributes to spasticity after spinal cord injury. Nat Med (2010) 16(3):302-7. doi:10.1038/nm. 2107

32. Kafitz KW, Rose CR, Thoenen H, Konnerth A. Neurotrophin-evoked rapid excitation through TrkB receptors. Nature (1999) 401(6756):918-21. doi:10.1038/ 44847

33. Constandil L, Aguilera R, Goich M, Hernández A, Alvarez P, Infante C, et al. Involvement of spinal cord BDNF in the generation and maintenance of chronic neuropathic pain in rats. Brain Res Bull (2011) 86(5-6):454-9. doi:10.1016/j.brainresbull.2011.08.008

34. Biggs JE, Lu VB, Stebbing MJ, Balasubramanyan S, Smith PA. Is BDNF sufficient for information transfer between microglia and dorsal horn neurons during the onset of central sensitization? Mol Pain (2010) 6:44. doi:10.1186/1744-80696- 44

35. Thompson CK, Jayaraman A, Kinnaird C, Hornby TG. Methods to quantify pharmacologically induced alterations in motor function in human incomplete SCI. J Vis Exp (2011) 50:e2148. doi:10.3791/2148

36. Wainberg M, Barbeau H, Gauthier S. The effects of cyproheptadine on locomotion and on spasticity in patients with spinal cord injuries. J Neurol Neurosurg Psychiatry (1990) 53(9):754-63. doi:10.1136/jnnp.53.9.754

37. Botte MJ, Nickel VL, Akeson WH. Spasticity and contracture. Physiologic aspects of formation. Clin Orthop Relat Res (1988) 233:7-18.

38. Donnelly DJ, Popovich PG. Inflammation and its role in neuroprotection, axonal regeneration and functional recovery after spinal cord injury. Exp Neurol (2008) 209:378-88. doi:10.1016/j.expneurol.2007.06.009

39. Gulino R, Lombardo SA, Casavona A, Leanza G, Perciavalle V. Level of brain-derived neurotrophic factor and neurotrophin-4 in lumbar motoneurons after low-thoracic spinal cord hemisection. Brain Res (2004) 1013:174-81. doi:10.1016/j.brainres.2004.03.055

40. Ikeda O, Murakami M, Ino H, Yamazaki M, Nemoto T, Koda M, et al. Acute up-regulation of brain-derived neurotrophic factor expression resulting from experimentally induced injury in the rat spinal cord. Acta Neuropathol (2001) 102:239-45.

41. Boyce VS, Park J, Gage FH, Mendell LM. Differential effects of brain-derived neurotrophic factor and neurotrophin-3 on hindlimb function in paraplegic rats. Eur J Neurosci (2012) 35(2):221-32. doi:10.1111/j.1460-9568.2011.07950.x

42. Gonzales M, Collins WF III. Modulation of motoneuron excitability by brainderived neurotrophic factor. J Neurophysiol (1997) 77:502-6.
43. Carvalho AL, Caldeira MV, Santos SD, Duarte CB. Role of the brain-derived neurotrophic factor at glutamatergic synapses. Br J Pharmacol (2007) 153(Suppl 1):S310-24. doi:10.1038/sj.bjp.0707509

44. Blum R, Konnerth A. Neurotrophin-mediated rapid signalling in the central nervous system: mechanisms and functions. Physiology (2005) 20:70-8. doi:10.1152/physiol.00042.2004

45. Rivera C, Voipio J, Thomas-Crusells J, Li H, Emri Z, Sipilä S, et al. Mechanism of activity-dependent downregulation of the neuron-specific K-Cl cotransporter KCC2. J Neurosci (2004) 24(19):4683-91. doi:10.1523/JNEUROSCI.5265-03. 2004

46. Vicario-Abejon C, Collin C, McKay RD, Segal M. Neurotrophins induce formation of functional excitatory and inhibitory synapses between cultured hippocampal neurons. J Neurosci (1998) 18:7256-71.

47. Rutherford LC, Nelson SB, Turrigiano GG. BDNF has opposite effects on the quantal amplitude of pyramidal neuron and interneuron excitatory synapses. Neuron (1998) 21(3):521-30. doi:10.1016/S0896-6273(00)80563-2

48. Bolton MM, Pittman AJ, Lo DC. Brain-derived neurotrophic factor differentially regulates excitatory and inhibitory synaptic transmission in hippocampal cultures. J Neurosci (2000) 20(9):3221-32.

49. Murray KC, Nakae A, Stephens MJ, Rank M, D’Amico J, Harvey PJ, et al. Recovery of motoneuron and locomotor function after spinal cord injury depends on constitutive activity in 5-HT2C receptors. Nat Med (2010) 16(6):694-700. doi: $10.1038 / \mathrm{nm} .2160$

50. Hounsgaard J, Hultborn H, Jespersen B, Kiehn O. Bistability of $\alpha$-motoneurones in the decerebrate cat and in the acute spinal cat after intravenous 5hydroxytryptophan. J Physiol (1988) 405:345-67.

Conflict of Interest Statement: The authors declare that the research was conducted in the absence of any commercial or financial relationships that could be construed as a potential conflict of interest.

Received: 02 August 2013; paper pending published: 04 September 2013; accepted: 04 November 2013; published online: 19 November 2013.

Citation: Fouad K, Bennett DJ, Vavrek R and Blesch A (2013) Long-term viral brainderived neurotrophic factor delivery promotes spasticity in rats with a cervical spinal cord hemisection. Front. Neurol. 4:187. doi: 10.3389/fneur.2013.00187

This article was submitted to Movement Disorders, a section of the journal Frontiers in Neurology.

Copyright (c) 2013 Fouad, Bennett, Vavrek and Blesch. This is an open-access article distributed under the terms of the Creative Commons Attribution License (CC BY). The use, distribution or reproduction in other forums is permitted, provided the original author(s) or licensor are credited and that the original publication in this journal is cited, in accordance with accepted academic practice. No use, distribution or reproduction is permitted which does not comply with these terms. 